\title{
Comparative Study of Efficacy of Topical Dapsone 5\% Gel, Topical Benzoyl Peroxide 2.5\% Alone and in Combination in Acne Vulgaris Patients
}

\author{
Dr.V.Lakshmi Sarojini ${ }^{1}$, Dr.S.Nageswaramma ${ }^{2}$, Dr.G.Sirisha ${ }^{3}$, Dr.N.K.Sagar ${ }^{4}$, \\ K.Mokshnanand ${ }^{5}$ \\ Department Of Dvl, Guntur Medical College, Ntruhs, Andhra Pradesh, India
}

\begin{abstract}
Acne is an extremely common and a complex skin disorder with a significant detrimental effect on the quality of life of the patients. The aim of this study is to assess the comparative efficacy of topical dapsone $5 \%$ gel and topical BPO 2.5\% alone and in combination in patients of acne over a 12 week period.Patients ith acne were selected randomly and divided into 3 groups in which one group received topical dapsone 5\% gel, econd group topical BPO 2.5\% and the third a combination of both. Grading of acne severity and laboratory investigations at baseline and at every two weeks were carried out. Significance testing was carried out with ppropriate statistical tests and $P$ values of $<0.05$ were considered significant. Out of 150 patients with grade 1,2,3 acne enrolled in the study ,50 patients were treated with dapsone alone out of which 45(90\%) patients with grade 1 and 2 acne showed $40 \%$ reduction in the number of inflammatory lesions. Among 50 patients who received BPO alone, $43(86 \%)$ showed $30 \%$ reduction in the inflammatory lesions and in the remaining 50 patients who received combination of dapsone and BPO 40(80\%) patients showed $50 \%$ reduction. So patients treated with a combination of dapsone and BPO showed better results than monotherapy.
\end{abstract}

Keywords: Acne, $B P O$, Dapsone ,topical

\section{Introduction}

Acne vulgaris is a complex multifactorial skin disorder involving multiple abnormalities of the pilosebaceous unit, including hyperkeratinisation, sebum production, bacterial proliferation and inflammation,influenced by harmones, food, stress etc.[1].Disease onset occurs commonly during adolescence and is characterised by non inflammatory lesions like comedones and inflammatory lesions like papules and pustules. Prevalence of acne is 39\% in the age group of 12 and $86 \%$ in the age group of 17

Dapsone is a sulfone with anti -inflammatory properties .Anti-inflammatory properties of dapsone include inhibition of neutrophil myeloperoxidase, scavenzing of reactive oxyzen species, decreasing prostaglandin and leukotriene release[2,3,4].Orally dapsone has been used in the past for recalcitrant acne(in the doses of $25-50 \mathrm{mg} / \mathrm{day}$ ) but haematological and other complications limit its use.It was hypothesised that a topical formulation of dapsone may be appropriate in treating acne vulgaris while minimising systemic exposure and haematological risk[5].Accordingly a topical gel formulation of dapsone 5\% was developed and approved by USFDA in 2005[6].The usage of dapsone gel has been tried as monotherapy, given twice daily and in combination with other topical modalities like benzoyl peroxide and adapalene.

The present study was undertaken to compare the efficacy of topical dapsone $5 \%$ gel with topical benzoyl peroxide (BPO) 2.5\% and with a combination of both topical dapsone 5\% gel and topical benzoyl peroxide $2.5 \%$ in acne vulgaris patients.

\section{Materials and Methods}

This is a prospective, randomized, open label, comparative therapeutic study conducted for a period of 4 months. All patients suffering from acne vulgaris between the ages of $15-45$ years were included in the study. Acne severity is assessed by global acne assessment score and acne grades 1,2,3 were included in this study. Laboratory investigations like Hb , TC , DC, ESR , RBS, LFT , RFT were carried out at baseline and two week interval. The study sample is divided in three groups in which one group was treated with topical dapsone $5 \%$ gel alone, second group with topical benzoyl peroxide $2.5 \%$ alone and the third group was treated with a combination of both topical dapsone 5\%gel and topical benzoyl peroxide 2.5\%.Patients who used other treatments for acne in the past 3 months, patients with haemoglobin less than $10 \mathrm{gm} \%$, grade 4 acne and pregnant women were excluded from the study 


\section{Results}

Out of 150 patients with grade 1, 2, 3 acne vulgaris enrolled in the study, 95 were males and 55 were females.Severity of acne in these patients was assessed by global acne assessment score and then were kept on the study drugs for a period of 12 weeks

\section{Table 1: Global acne assessment score}

\begin{tabular}{|l|l|l|}
\hline 0 & None & No evidence of facial acne vulgaris \\
\hline I & Minimal & $\begin{array}{l}\text { Few non inflammatory lesions (comedones) are present; a few inflammatory } \\
\text { lesions (papules / pustules) may be present. }\end{array}$ \\
\hline II & Mild & $\begin{array}{l}\text { Several to many non- inflammatory lesions (comedones) are present ; a few } \\
\text { inflammatory lesions(papules/pustules) are present. }\end{array}$ \\
\hline III & Moderate & $\begin{array}{l}\text { Many non -inflammatory (comedones) and inflammatory lesions } \\
\text { (papules/pustules) are present ; no nodulo-cystic lesions are allowed. }\end{array}$ \\
\hline IV & Severe & $\begin{array}{l}\text { Significant degree of inflammatory disease; papules/pustules are predominant } \\
\text { feature; few nodulo-cystic lesions may be present; comedones may be present. }\end{array}$ \\
\hline
\end{tabular}

Among the study sample , 40(26.66\%) patients were of grade 1,88(58.66\%) were of garde 2 and $22(14.66 \%)$ patients were of grade 3 acne. During the study period, dapsone gel alone was received by 50 patients where $33(66 \%)$ were males and $17(34 \%)$ were females. Among this group , 13(26\%) were grade 1 , $32(64 \%)$ grade $2,5(10 \%)$ were grade 3 . Second group of 50 patients received benzoyl peroxide alone . among these patients $34(68 \%)$ were males and $16(32 \%)$ were females.15(30\%) had grade 1 acne , 28(56\%) had grade 2 and $7(14 \%)$ had grade 3 acne. 50 patients received a combination of dapsone and benzoyl peroxide, among them $28(56 \%)$ were males and 22(44\%) were females.Grade 1 acne were noted in $12(24 \%)$, grade 2 in $28(56 \%)$ and grade 3 acne in $10(20 \%)$.(Table 2$)$

Table 2

\begin{tabular}{|l|l|l|l|}
\hline Sex & $\begin{array}{l}\text { Dapsone 5\%6 gel alone } \\
\text { n-50 }\end{array}$ & $\begin{array}{l}\text { Benzoy! peroxide } \\
2.5 \%\end{array}$ & $\begin{array}{l}\text { Dapsone gel + benzoyt } \\
\text { peroxide } n=50\end{array}$ \\
\hline Males & $33(60 \%)$ & $34(68 \%)$ & $28(56 \%)$ \\
\hline females & $17(34 \%)$ & $16(32 \%)$ & $22(44 \%)$ \\
\hline
\end{tabular}

\begin{tabular}{|l|l|l|l|}
\hline $\begin{array}{l}\text { GAAS Score at } \\
\text { baseline }\end{array}$ & & & \\
\hline Grade 1 & $13(26 \%)$ & $15(30 \%)$ & $12(24 \%)$ \\
\hline Grade 2 & $32(64 \%)$ & $28(56 \%)$ & $28(56 \%)$ \\
\hline Grade 3 & $5(10 \%)$ & $7(14 \%)$ & $10(20 \%)$ \\
\hline
\end{tabular}

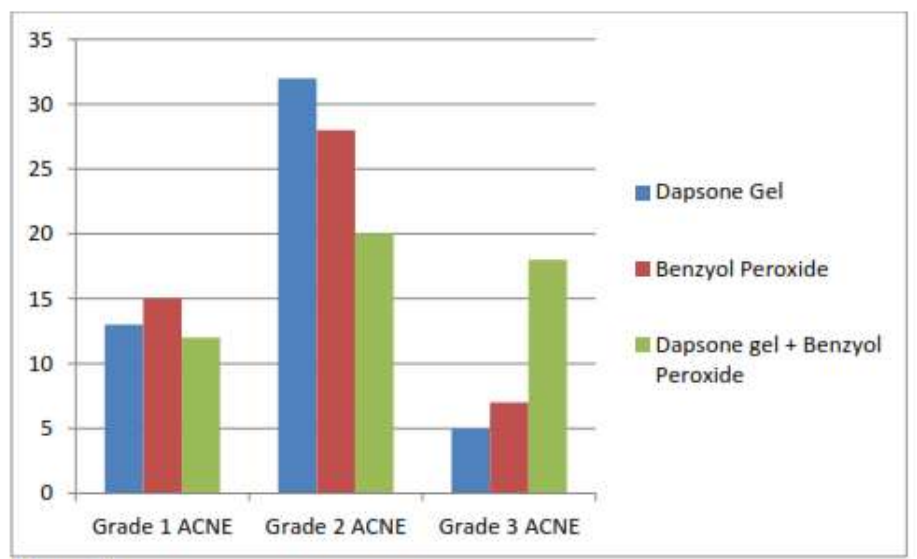

Figure 1 
Out of 50 patients who were treated with dapsone gel alone 45(90\%) patients with grade 1 and 2 acne responded well with $40 \%$ reduction in the number of inflammatory lesions within 2 weeks. $5(10 \%)$ grade 3 acne patients had no response. Results were noted 2 weeks apart. Out of 50 patients treated with BPO alone 43(86\%) patients with grade 1 and 2 acne responded well with 30\% reduction in the number of inflammatory lesions within 2 weeks.Patients with grade 3 acne i.e 7(14\%) did not respond. Among the 50 patients who received both dapsone and BPO 40(80\%) patients with grade 1 and 2 acne responded well with 50\% reduction in the inflammatory lesions within 2 weeks which is higher when compared to either of the drugs alone.10(20\%) patients with grade 3 acne also responded well with $30 \%$ reduction in inflammatory lesions where no response was noted with either of the drugs alone.

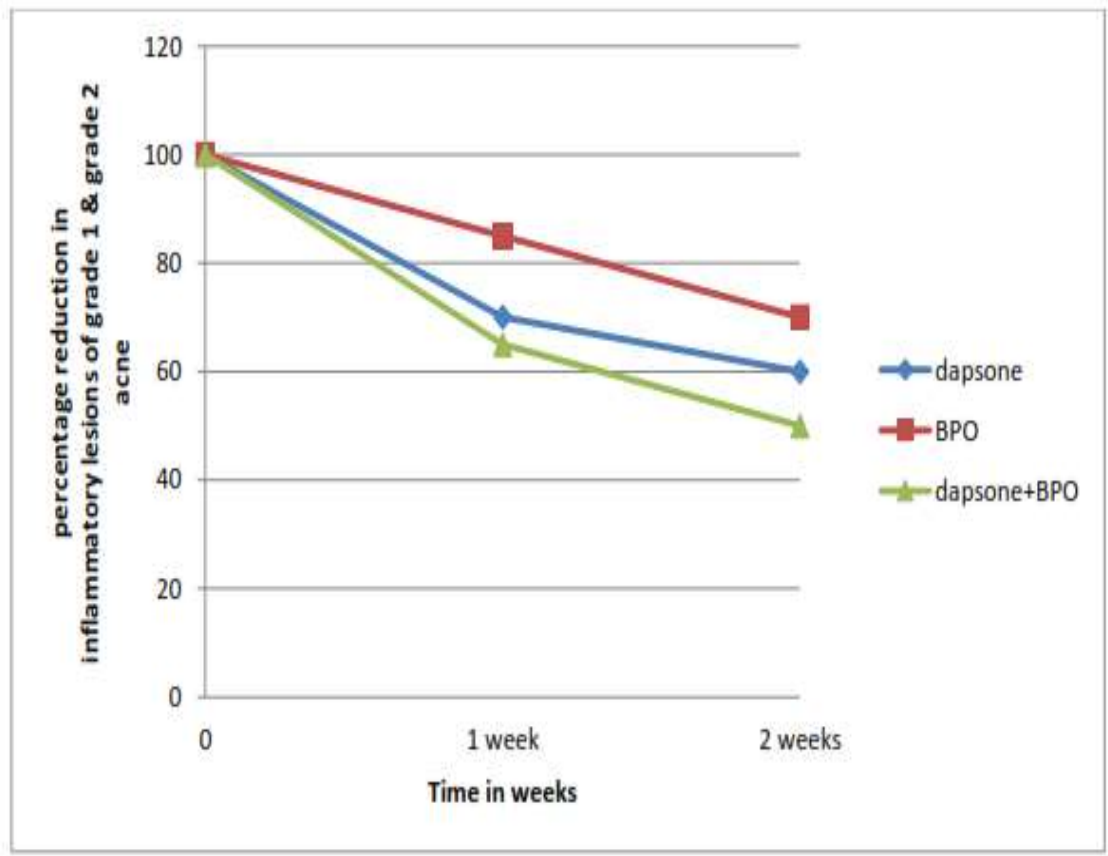

Figure 2

3.1Statistical analysis: Statistical analysis in this study was carried out using Chi-square test to test the significance difference between the drugs i.e dapsone gel, benzoyl peroxide and a combination of dapsone gel and benzoyl peroxide. Although the clinical response showed that the dapsone gel monotherapy is efficacious than BPO monotherapy, statistically we found that there is no significance difference with $\mathrm{p}$ value is $<0.05$. combination therapy with BPO and dapsone gel has better response than monotherapy both clinically and statistically significant with $\mathrm{p}$ value $>0.05$.

Acne vulgaris is the most common skin disease of adolescents and young adults with a reported prevalence being nearly $80 \%$ [7].Its predominance in this psychologically labile age group together with its potentially life long sequelae makes it a matter of huge financial and psychosocial concern.

There is no ideal treatment for acne, although a suitable regimen targetted at reducing the number of lesions and preventing the permanent sequelae, can be made out for most of the patients.The current standard treatment approach of acne vulgaris is targetted towards the type of lesions and severity of acne[8]. The high efficacy of dapsone and compatibility with other concurrent acne medications makes topical dapsone a key component in the treatment of acne[9].

Dapsone is a sulfone with both anti-inflammatory and antimicrobial properties. It has been available for over 60 years and has been used to treat myriad of cutaneous disordes like leprosy, dermatitis herpetiformis etc .The potential of oral dapsone to treat acne vulgaris is well established, but the risks of serious side effects have made it undesirable for the use in relatively healthy acne population.

The oral formulation of dapsone is known to cause haematological adverse effects including dose-dependent hemolysis due to oxidative damage to red blood cells from its hydroxylamine metabolite[10,11]. Individuals with glucose-6-phosphate dehydrogenase deficiency are more sensitive to haemolytic anemia after exposure to stressors including dapsone[12].

A topical formulation of dapsone was recently developed to deliver therapeutic concentrations of dapsone to the skin. Clinical studies have shown that dapsone gel $5 \%$ was effective in the treatment of acne 
vulgaris[5] with approximately $1 \%$ of the systemic exposure that is seen with typical oral dapsone treatment $[13,14]$. After treatment with dapsone gel 5\%, no clinical or lab evidence of drug induced hemolytic anaemia was noted in G6PD deficient subjects with acne vulgaris[15].

In two double blind, randomised, 12 week, vehicle controlled studies[5], significantly better outcomes were observed for patients applying dapsone gel versus the vehicle. The cutaneous side effects noticed are facial oiliness, peeling, dryness, erythema and itching which does not interfere with the completion of the treatment.

In a study by Thiboutot DM et al, to report the pharmacokinetic profile of topically applied dapsone gel $5 \%$ in the treatment of acne vulgaris it was concluded that topical application of dapsone gel in various settings ranging from 2 weeks to 12 months resulted in systemic exposures to dapsone and its metabolites that were approximately 100 fold less than those after oral dapsone at therapeutic dose level. The concentration of dapsone and its metabolites reached steady state and did not increase during prolonged treatment.

Topical dapsone $5 \%$ gel offers documented efficacy for the reduction of inflammatory lesions of mild to moderate acne especially in patients exhibiting intolerance to conventional anti acne agents[14,15]. Most of the anti-acne medications do not act on all the pathophysiological features of acne, so combination therapy is often used in the management[16-18]. It has been evaluated in combination with adapalene $0.1 \%$ as well as BPO $4 \%$ and has been reported to be safe and well tolerated[19]. dose-dependent hemolysis due to oxidative damage to red blood cells from its hydroxylamine metabolite[10,11]. Individuals with glucose-6-phosphate dehydrogenase deficiency are more sensitive to haemolytic anemia after exposure to stressors including dapsone[12].

A topical formulation of dapsone was recently developed to deliver therapeutic concentrations of dapsone to the skin. Clinical studies have shown that dapsone gel 5\% was effective in the treatment of acne vulgaris[5] with approximately $1 \%$ of the systemic exposure that is seen with typical oral dapsone treatment $[13,14]$. After treatment with dapsone gel 5\%, no clinical or laboratory evidence of drug induced hemolytic anaemia was noted in G6PD deficient subjects with acne vulgaris[15].

In two double blind, randomised, 12 week, vehicle controlled studies[5], significantly better outcomes were observed for patients applying dapsone gel versus the vehicle. The cutaneous side effects noticed are facial oiliness, peeling, dryness, erythema and itching which does not interfere with the completion of the treatment.

In a study by Thiboutot DM et al, to report the pharmacokinetic profile of topically applied dapsone gel 5\% in the treatment of acne vulgaris it was concluded that topical application of dapsone gel in various settings ranging from 2 weeks to 12 months resulted in systemic exposures to dapsone and its metabolites that were approximately 100 fold less than those after oral dapsone at therapeutic dose level. The concentration of dapsone and its metabolites reached steady state and did not increase during prolonged treatment.

Topical dapsone $5 \%$ gel offers documented efficacy for the reduction of inflammatory lesions of mild to moderate acne especially in patients exhibiting intolerance to conventional anti acne agents[14,15]. Most of the anti-acne medications do not act on all the pathophysiological features of acne, so combination therapy is often used in the management[16-18]. It has been evaluated in combination with adapalene $0.1 \%$ as well as BPO $4 \%$ and has been reported to be safe and well tolerated[19].

The present study was carried out to assess and compare the efficacy of topical dapsone 5\% gel , topical benzoyl peroxide $2.5 \%$, and the combination of these two drugs in patients suffering from acne vulgaris. In all the three treatment groups improvements in acne were observed as early as 2 weeks. Better response , measured by percentage reduction of inflammatory lesions from the baseline was observed with dapsone gel. Further improvement was observed for patients who received dapsone gel in combination with BPO. The results were consistent with previous controlled trails.

Discontinuation of the study due to treatment-related adverse events was not observed. Local adverse reactions were minimal, mild in severity and they improved during treatment. No systemic adverse effects were observed.

\section{Conclusion}

With this study we would like to conclude that dapsone gel 5\% is efficaious and can be safely used for acne patients with inflammatory lesions and who are intolerable to other treatment modalities. Combination of dapsone gel with BPO can be safely used in acne patients with better results in grade 3 acne patients

\section{References}

[1]. Pawin H , BeylotC, ChivotM , et al. Physiopathology of acne vulgaris : recent data, new understanding of the treatments. Eur $\mathbf{J}$ Dermatol . 2004:14(1):4-12.

[2]. Zhuyl, StillesMJ. Dapsone and sulfones in dermatology review update. J.AmAcad.Dermatol 2001;45:420-434

[3]. Wozel G, Barth J.current aspects of modes of action of dapsone.Intl.J.Dermatol 1988;27:1547552 
[4]. Stotland M, shalita AR, Kissling RF. Dapsone 5\% gel; a review of its efficacy and safety in therapy of acne vulgaris. Am.J.Clin.Dermatol 2009; 10:221-227

[5]. Draclos D, Carter EMaloney JM et al.Two randomized studies to demonstrate the efficacy and safety of dapsone gel 5\% for the treatment of acne vulgaris , J Am . Acad. Dermatol: 2007; 56(3);439.e1-10

[6]. PickertA An evaluation of dapsone gel 5\% in the treatment of A.V expert spin . Pharmacoether 20009; 10(9): 1515-1521

[7]. Cunliffe WJ , Gould DJ. Prevalence of facial acne vulgaris in late adolescence and in adults. Br.Med J1979;1:1109-10

[8]. Hayash N , Akamatsu H , Kawashima M , Acne study group. Establishment of grading criteria for acne severity. J Dermatol 2007;35:255-60

[9]. Sashikumar BM topical dapsone -A novel approach in A.V. Intl . Journal of universal pharmacy and life science 2012, 2(2), 74-79.

[10]. Beutler E G6PD deficiency, Blood 1994;84(11)3613-3636.

[11]. Thiboutot DM Willmer Jsharata HHalder RGarrett S Pharmacokinetics of dapsone gel $5 \%$ for the treatment of acne vulgaris.Clin Pharmacokinet 2007;46(8)697-712

[12]. Lucky AW Maloney JM Roberts J et al. Dapsone gel 5\% for the treatment of acne vulgaris; safety and efficacy of long term (1year)treatment. J Drugs Dermatol 2007;6(10) 981-987.

[13]. Piette ww. Haematologic safety of dapsone gel, 5\% for topical treatment of A.V . Arch. Dermatl.2008 144(12) 1564-1570.

[14]. Tan J Dapsone 5\% gel:A new option in topical therapy for acne.Skin therapy Lett 2012;17:1-3.

[15]. Kircik LH.Harnessing the anti -inflammatory effects of topical dapsone for the management of acne.J Drugs Dermatol 2010; 9:66771.

[16]. Gollnick H, Cunliffe W, Berson D, et al; and Global Alliance to improve outcomes in Acne..Management of acne: A report from a Global alliance to improve outcomes of acne.J Am Acad Dermatol $2003 ; 49$ (1 suppl):1S-37S.

[17]. Strauss JS , Krowchuk DP , Leyden JJ , et al; American Academy of Dermatology /American Academy of dermatology associatin.Guidelines of care of acne vulgaris management.J AM Acad Dermatol 2007;56(4):651-663.

[18]. Leyden JJ.Areview of the use of combination therapies for the treatment of acne vulgaris. J AM Acad Dermatol 2003;49(3 suppl) S200-S210.

[19]. Fleischer AB Jr . Et al -dapsone gel $5 \%$ in combination with adapalene gel $0.1 \%$, benzoyl per oxide gel $4 \%$ or moisterizer for treatment of acne vulgaris: a12 week randomized double blind study . J drugs dermatology 2010, 9(1), 33-40. 